\title{
SOME MULTIPLICATIVE INEQUALITIES FOR HEINZ OPERATOR MEAN
}

\author{
SILVESTRU SEVER DRAGOMIR
}

\begin{abstract}
In this paper we obtain some new multiplicative inequalities for Heinz operator mean..

MSC(2010): 47A63, 47A30, 15A60, 26D15, 26D10.

Keywords: Young's Inequality, Real functions, Arithmetic mean-Geometric mean inequality, Heinz means.
\end{abstract}

\section{Introduction}

Throughout this paper $A, B$ are positive invertible operators on a complex Hilbert space $(H,\langle\cdot, \cdot\rangle)$. We use the following notations for operators and $\nu \in[0,1]$

$$
A \nabla_{\nu} B:=(1-\nu) A+\nu B
$$

the weighted operator arithmetic mean, and

$$
A \sharp_{\nu} B:=A^{1 / 2}\left(A^{-1 / 2} B A^{-1 / 2}\right)^{\nu} A^{1 / 2},
$$

the weighted operator geometric mean [14]. When $\nu=\frac{1}{2}$ we write $A \nabla B$ and $A \sharp B$ for brevity, respectively.

Define the Heinz operator mean by

$$
H_{\nu}(A, B):=\frac{1}{2}\left(A \sharp_{\nu} B+A \sharp_{1-\nu} B\right) \text {. }
$$

The following interpolatory inequality is obvious

$$
A \sharp B \leq H_{\nu}(A, B) \leq A \nabla B
$$

for any $\nu \in[0,1]$.

We recall that Specht's ratio is defined by [16]

$$
S(h):=\left\{\begin{array}{l}
\frac{h^{\frac{1}{h-1}}}{e \ln \left(h^{\frac{1}{h-1}}\right)} \text { if } h \in(0,1) \cup(1, \infty), \\
1 \text { if } h=1 .
\end{array}\right.
$$

Date: Received: October 8, 2020, Accepted: January 11, 2021. 
It is well known that $\lim _{h \rightarrow 1} S(h)=1, S(h)=S\left(\frac{1}{h}\right)>1$ for $h>0, h \neq 1$. The function is decreasing on $(0,1)$ and increasing on $(1, \infty)$.

The following result provides an upper and lower bound for the Heinz mean in terms of the operator geometric mean $A \sharp B$ :

Theorem 1.1 (Dragomir, 2015 [6]). Assume that A, $B$ are positive invertible operators and the constants $M>m>0$ are such that

$$
m A \leq B \leq M A \text {. }
$$

Then we have

$$
\omega_{\nu}(m, M) A \sharp B \leq H_{\nu}(A, B) \leq \Omega_{\nu}(m, M) A \sharp B,
$$

where

$$
\Omega_{\nu}(m, M):=\left\{\begin{array}{l}
S\left(m^{|2 \nu-1|}\right) \text { if } M<1, \\
\max \left\{S\left(m^{|2 \nu-1|}\right), S\left(M^{|2 \nu-1|}\right)\right\} \quad \text { if } m \leq 1 \leq M, \\
S\left(M^{|2 \nu-1|}\right) \text { if } 1<m
\end{array}\right.
$$

and

$$
\omega_{\nu}(m, M):=\left\{\begin{array}{l}
S\left(M^{\left|\nu-\frac{1}{2}\right|}\right) \text { if } M<1, \\
1 \text { if } m \leq 1 \leq M, \\
S\left(m^{\left|\nu-\frac{1}{2}\right|}\right) \text { if } 1<m,
\end{array}\right.
$$

where $\nu \in[0,1]$.

We consider the Kantorovich's constant defined by

$$
K(h):=\frac{(h+1)^{2}}{4 h}, h>0 .
$$

The function $K$ is decreasing on $(0,1)$ and increasing on $[1, \infty), K(h) \geq 1$ for any $h>0$ and $K(h)=K\left(\frac{1}{h}\right)$ for any $h>0$.

We have:

Theorem 1.2 (Dragomir, 2015 [7]). Assume that A, B are positive invertible operators and the constants $M>m>0$ are such that the condition (1.3) is valid. Then for any $\nu \in[0,1]$ we have

$$
(A \sharp B \leq) H_{\nu}(A, B) \leq \exp \left[\Theta_{\nu}(m, M)-1\right] A \sharp B
$$

where

$$
\Theta_{\nu}(m, M):=\left\{\begin{array}{l}
K\left(m^{|2 \nu-1|}\right) \text { if } M<1, \\
\max \left\{K\left(m^{|2 \nu-1|}\right), K\left(M^{|2 \nu-1|}\right)\right\} \text { if } m \leq 1 \leq M, \\
K\left(M^{|2 \nu-1|}\right) \text { if } 1<m
\end{array}\right.
$$


and

$$
(0 \leq) H_{\nu}(A, B)-A \sharp B \leq \frac{1}{4 m^{1-\nu}} \max _{x \in[m, M]} D\left(x^{2 \nu-1}\right) A,
$$

where the function $D:(0, \infty) \rightarrow[0, \infty)$ is defined by $D(x)=(x-1) \ln x$.

The following bounds for the Heinz mean $H_{\nu}(A, B)$ in terms of $A \nabla B$ are also valid:

Theorem 1.3 (Dragomir, 2015 [7]). With the assumptions of Theorem 2.2 we have

$$
(0 \leq) A \nabla B-H_{\nu}(A, B) \leq \nu(1-\nu) \Upsilon(m, M) A,
$$

where

$$
\Upsilon(m, M):=\left\{\begin{array}{l}
(m-1) \ln m \text { if } M<1, \\
\max \{(m-1) \ln m,(M-1) \ln M\} \text { if } m \leq 1 \leq M, \\
(M-1) \ln M \text { if } 1<m
\end{array}\right.
$$

and

$$
A \nabla B \exp [-4 \nu(1-\nu)(\digamma(m, M)-1)] \leq H_{\nu}(A, B)(\leq A \nabla B)
$$

where

$$
\digamma(m, M):=\left\{\begin{array}{l}
K(m) \text { if } M<1, \\
\max \{K(m), K(M)\} \text { if } m \leq 1 \leq M, \\
K(M) \text { if } 1<m .
\end{array}\right.
$$

For other recent results on operator geometric mean inequalities, see [2][13], [15] and [17]-[18].

Motivated by the above results, we establish in this paper some multiplicative inequalities providing bounds for $H_{\nu}(A, B)$ in terms of $A \sharp B$ and $A \nabla B$ under various assumptions for positive invertible operators $A, B$.

\section{Bounds for $H_{\nu}(A, B)$ in Terms of $A \sharp B$}

For $\nu \in(0,1) \backslash\left\{\frac{1}{2}\right\}$ we consider the following function $d_{\nu}:(0, \infty) \rightarrow[1, \infty)$ defined by

$$
d_{\nu}(x)=\frac{x^{\nu}+x^{1-\nu}}{2 \sqrt{x}} .
$$

The properties of this function are collected in the following lemma.

Lemma 2.1. For any $\nu \in(0,1) \backslash\left\{\frac{1}{2}\right\}$ we have that $\lim _{x \rightarrow 0+} d_{\nu}(x)=$ $\lim _{x \rightarrow \infty} d_{\nu}(x)=\infty$, the function is decreasing on $(0,1)$, increasing on $(1, \infty), d_{\nu}(1)=1$ and $d_{\nu}\left(\frac{1}{x}\right)=d_{\nu}(x)$ for any $x \in(0, \infty)$. 
Proof. We have

$$
d_{\nu}(x)=\frac{x^{\nu}+x^{1-\nu}}{2 \sqrt{x}}=\frac{1}{2}\left(x^{\nu-\frac{1}{2}}+x^{\frac{1}{2}-\nu}\right)
$$

for any $x \in(0, \infty)$ and any $\nu \in(0,1) \backslash\left\{\frac{1}{2}\right\}$.

By taking the derivative we have

$$
\begin{aligned}
d_{\nu}^{\prime}(x) & =\frac{1}{2}\left(\left(\nu-\frac{1}{2}\right) x^{\nu-\frac{3}{2}}+\left(\frac{1}{2}-\nu\right) x^{-\nu-\frac{1}{2}}\right) \\
& =\frac{1}{2}\left(\nu-\frac{1}{2}\right)\left(x^{\nu-\frac{3}{2}}-x^{-\nu-\frac{1}{2}}\right) \\
& =\frac{1}{2}\left(\nu-\frac{1}{2}\right) x^{-\nu-\frac{1}{2}}\left(x^{2 \nu-1}-1\right)
\end{aligned}
$$

for any $x \in(0, \infty)$ and any $\nu \in(0,1) \backslash\left\{\frac{1}{2}\right\}$.

If $\nu>\frac{1}{2}$ then $x^{2 \nu-1}-1$ is negative for $x \in(0,1)$ and positive for $x \in(1, \infty)$ giving that $d_{\nu}^{\prime}(x)$ is negative for $x \in(0,1)$ and positive for $x \in(1, \infty)$.

If $\nu<\frac{1}{2}$ then $x^{2 \nu-1}-1$ is positive for $x \in(0,1)$ and negative for $x \in(1, \infty)$ giving that $d_{\nu}^{\prime}(x)$ is negative for $x \in(0,1)$ and positive for $x \in(1, \infty)$.

These imply that $d_{\nu}$ is decreasing on $(0,1)$ and increasing on $(1, \infty)$. The rest is obvious.

Theorem 2.2. Let $A, B$ be positive invertible operators and the constants $M>m>0$ such that

$$
m A \leq B \leq M A .
$$

If for $\nu \in(0,1) \backslash\left\{\frac{1}{2}\right\}$ we define

$$
\Lambda_{\nu}(m, M):=\left\{\begin{array}{l}
\frac{m^{\nu}+m^{1-\nu}}{2 \sqrt{m}} \text { if } M<1, \\
\max \left\{\frac{m^{\nu}+m^{1-\nu}}{2 \sqrt{m}}, \frac{M^{\nu}+M^{1-\nu}}{2 \sqrt{M}}\right\} \text { if } m \leq 1 \leq M, \\
\frac{M^{\nu}+M^{1-\nu}}{2 \sqrt{M}} \text { if } 1<m
\end{array}\right.
$$

and

$$
\lambda_{\nu}(m, M):=\left\{\begin{array}{l}
\frac{M^{\nu}+M^{1-\nu}}{2 \sqrt{M}} \text { if } M<1, \\
1 \text { if } m \leq 1 \leq M \\
\frac{m^{\nu}+m^{1-\nu}}{2 \sqrt{m}} \text { if } 1<m
\end{array}\right.
$$

then we have the double inequality

$$
\lambda_{\nu}(m, M) A \sharp B \leq H_{\nu}(A, B) \leq \Lambda_{\nu}(m, M) A \sharp B,
$$

for $\nu \in(0,1) \backslash\left\{\frac{1}{2}\right\}$. 
Proof. By the properties of function $d_{\nu}$ we have

$$
\begin{aligned}
& \left\{\begin{array}{l}
d_{\nu}(M) \text { if } M<1, \\
1 \text { if } m \leq 1 \leq M, \quad \leq \frac{x^{\nu}+x^{1-\nu}}{2 \sqrt{x}} \\
d_{\nu}(m) \text { if } 1<m,
\end{array}\right. \\
& \leq\left\{\begin{array}{l}
d_{\nu}(m) \text { if } M<1 \\
\max \left\{d_{\nu}(m), d_{\nu}(M)\right\} \text { if } m \leq 1 \leq M \\
d_{\nu}(M) \text { if } 1<m
\end{array}\right.
\end{aligned}
$$

for any $x \in[m, M]$ and any $\nu \in(0,1) \backslash\left\{\frac{1}{2}\right\}$.

This is equivalent to

$$
\lambda_{\nu}(m, M) \sqrt{x} \leq \frac{x^{\nu}+x^{1-\nu}}{2} \leq \Lambda_{\nu}(m, M) \sqrt{x}
$$

for any $x \in[m, M]$ and any $\nu \in(0,1) \backslash\left\{\frac{1}{2}\right\}$.

Using the continuous functional calculus, we have for any operator $X$ with $m I \leq X \leq M I$ that

$$
\lambda_{\nu}(m, M) X^{1 / 2} \leq \frac{X^{\nu}+X^{1-\nu}}{2} \leq \Lambda_{\nu}(m, M) X^{1 / 2}
$$

for any $\nu \in(0,1) \backslash\left\{\frac{1}{2}\right\}$.

Now, if we multiply both sides of (2.2) by $A^{-1 / 2}$ we have $m I \leq A^{-1 / 2} B A^{-1 / 2} \leq$ $M I$ and by writing the inequality (2.7) for $X=A^{-1 / 2} B A^{-1 / 2}$ we get

$$
\begin{aligned}
\lambda_{\nu}(m, M)\left(A^{-1 / 2} B A^{-1 / 2}\right)^{1 / 2} & \leq \frac{\left(A^{-1 / 2} B A^{-1 / 2}\right)^{\nu}+\left(A^{-1 / 2} B A^{-1 / 2}\right)^{1-\nu}}{2} \\
& \leq \Lambda_{\nu}(m, M)\left(A^{-1 / 2} B A^{-1 / 2}\right)^{1 / 2}
\end{aligned}
$$

for any $\nu \in(0,1) \backslash\left\{\frac{1}{2}\right\}$.

Finally, if we multiply both sides of (2.8) by $A^{1 / 2}$, then we get the desired result (2.5).

Corollary 2.3. Let $A, B$ be two positive operators. For positive real numbers $m, m^{\prime}, M, M^{\prime}$, put $h:=\frac{M}{m}, h^{\prime}:=\frac{M^{\prime}}{m^{\prime}}$ and let $\nu \in(0,1) \backslash\left\{\frac{1}{2}\right\}$. If either of the following conditions

(i) If $0<m I \leq A \leq m^{\prime} I<M^{\prime} I \leq B \leq M I$,

(ii) If $0<m I \leq B \leq m^{\prime} I<M^{\prime} I \leq A \leq M I$, hold, then

$$
\frac{\left(h^{\prime}\right)^{\nu}+\left(h^{\prime}\right)^{1-\nu}}{2 \sqrt{h^{\prime}}} A \sharp B \leq H_{\nu}(A, B) \leq \frac{h^{\nu}+h^{1-\nu}}{2 \sqrt{h}} A \sharp B .
$$


Proof. If the condition (i) is valid, then we have

$$
I<h^{\prime} I=\frac{M^{\prime}}{m^{\prime}} I \leq A^{-1 / 2} B A^{-1 / 2} \leq \frac{M}{m} I=h I,
$$

which implies, by (2.5) that

$$
d_{\nu}\left(h^{\prime}\right) A \sharp B \leq H_{\nu}(A, B) \leq d_{\nu}(h) A \sharp B
$$

and the inequality (2.9) is proved.

If the condition (ii) is valid, then we have

$$
0<\frac{1}{h} I \leq A^{-1 / 2} B A^{-1 / 2} \leq \frac{1}{h^{\prime}} I<I
$$

which, by (2.5) gives

$$
d_{\nu}\left(\frac{1}{h^{\prime}}\right) A \sharp B \leq H_{\nu}(A, B) \leq d_{\nu}\left(\frac{1}{h}\right) A \sharp B .
$$

Since

$$
d_{\nu}\left(\frac{1}{h^{\prime}}\right)=d_{\nu}\left(h^{\prime}\right) \text { and } d_{\nu}\left(\frac{1}{h}\right)=d_{\nu}(h),
$$

then the inequality (2.9) is also valid.

\section{Bounds for $H_{\nu}(A, B)$ in Terms of $A \nabla B$}

We introduce the function $c_{\nu}:(0, \infty) \rightarrow[1, \infty)$ defined by

$$
c_{\nu}(x)=\frac{x+1}{x^{\nu}+x^{1-\nu}}
$$

where $\nu \in(0,1) \backslash\left\{\frac{1}{2}\right\}$.

The properties of this function are as follows:

Lemma 3.1. For any $\nu \in(0,1) \backslash\left\{\frac{1}{2}\right\}$ we have that $\lim _{x \rightarrow 0+} c_{\nu}(x)=$ $\lim _{x \rightarrow \infty} c_{\nu}(x)=\infty$, the function is decreasing on $(0,1)$, increasing on $(1, \infty), c_{\nu}(1)=1$ and $c_{\nu}\left(\frac{1}{x}\right)=c_{\nu}(x)$ for any $x \in(0, \infty)$.

Proof. Taking the derivative of $c_{\nu}$, we have

$$
\begin{aligned}
c_{\nu}^{\prime}(x) & =\frac{(x+1)^{\prime}\left(x^{\nu}+x^{1-\nu}\right)-(x+1)\left(x^{\nu}+x^{1-\nu}\right)^{\prime}}{\left(x^{\nu}+x^{1-\nu}\right)^{2}} \\
& =\frac{x^{\nu}+x^{1-\nu}-(x+1)\left(\nu x^{\nu-1}+(1-\nu) x^{-\nu}\right)}{\left(x^{\nu}+x^{1-\nu}\right)^{2}} \\
& =\frac{x^{\nu}+x^{1-\nu}-\nu x^{\nu}-(1-\nu) x^{1-\nu}-\nu x^{\nu-1}-(1-\nu) x^{-\nu}}{\left(x^{\nu}+x^{1-\nu}\right)^{2}} \\
& =\frac{(1-\nu) x^{\nu}+\nu x^{1-\nu}-\nu x^{\nu-1}-(1-\nu) x^{-\nu}}{\left(x^{\nu}+x^{1-\nu}\right)^{2}} \\
& =\frac{(1-\nu)\left(x^{\nu}-x^{-\nu}\right)+\nu\left(x^{1-\nu}-x^{\nu-1}\right)}{\left(x^{\nu}+x^{1-\nu}\right)^{2}}
\end{aligned}
$$


for any $x \in(0, \infty)$ and $\nu \in(0,1) \backslash\left\{\frac{1}{2}\right\}$.

Consider the function $\ell_{\nu}:(0, \infty) \rightarrow \mathbb{R}$ defined by

$$
\begin{aligned}
\ell_{\nu}(x) & :=(1-\nu)\left(x^{\nu}-x^{-\nu}\right)+\nu\left(x^{1-\nu}-x^{\nu-1}\right) \\
& =(1-\nu)\left(x^{\nu}-\frac{1}{x^{\nu}}\right)+\nu\left(x^{1-\nu}-\frac{1}{x^{1-\nu}}\right) \\
& =(1-\nu)\left(\frac{x^{2 \nu}-1}{x^{\nu}}\right)+\nu\left(\frac{x^{2(1-\nu)}-1}{x^{1-\nu}}\right) .
\end{aligned}
$$

We also have

$$
\begin{aligned}
\ell_{\nu}^{\prime}(x) & =(1-\nu)\left(\nu x^{\nu-1}+\nu x^{-\nu-1}\right)+\nu\left((1-\nu) x^{-\nu}+(1-\nu) x^{\nu-2}\right) \\
& =(1-\nu) \nu\left(x^{\nu-1}+x^{-\nu-1}+x^{-\nu}+x^{\nu-2}\right)
\end{aligned}
$$

for any $x \in(0, \infty)$ and $\nu \in(0,1) \backslash\left\{\frac{1}{2}\right\}$.

Since $\ell_{\nu}^{\prime}(x)>0$ for any $x \in(0, \infty)$ and $\nu \in(0,1) \backslash\left\{\frac{1}{2}\right\}$ it follows that the equation $\ell_{\nu}(x)=0$ has a unique solution on $(0, \infty)$, namely $x=1$ and $\ell_{\nu}^{\prime}(x)<0$ for $x \in(0,1)$ and $\ell_{\nu}^{\prime}(x)>0$ for $x \in(1, \infty)$.

These show that the function $c_{\nu}$ is decreasing on $(0,1)$ and increasing on $(1, \infty)$.

The rest of properties are obvious.

We have:

Theorem 3.2. Let $A, B$ be positive invertible operators and the constants $M>m>0$ such that the condition (2.2) holds. If for $\nu \in(0,1) \backslash\left\{\frac{1}{2}\right\}$ we define

$$
\Phi_{\nu}(m, M):=\left\{\begin{array}{l}
\frac{M^{\nu}+M^{1-\nu}}{M+1} \text { if } M<1, \\
1 \text { if } m \leq 1 \leq M \\
\frac{m^{\nu}+m^{1-\nu}}{m+1} \text { if } 1<m,
\end{array}\right.
$$

and

$$
\phi_{\nu}(m, M):=\left\{\begin{array}{l}
\frac{m^{\nu}+m^{1-\nu}}{m+1} \text { if } M<1, \\
\min \left\{\frac{m^{\nu}+m^{1-\nu}}{m+1}, \frac{M^{\nu}+M^{1-\nu}}{M+1}\right\} \text { if } m \leq 1 \leq M, \\
\frac{M^{\nu}+M^{1-\nu}}{M+1} \text { if } 1<m,
\end{array}\right.
$$

then we have the double inequality

$$
\phi_{\nu}(m, M) A \nabla B \leq H_{\nu}(A, B) \leq \Phi_{\nu}(m, M) A \nabla B,
$$

for $\nu \in(0,1) \backslash\left\{\frac{1}{2}\right\}$. 
Proof. From Lemma 3.1 we have

$$
\begin{aligned}
& \left\{\begin{array}{l}
\frac{M+1}{M^{\nu}+M^{1-\nu}} \text { if } M<1, \\
1 \text { if } m \leq 1 \leq M, \\
\frac{m+1}{m^{\nu}+m^{1-\nu}} \text { if } 1<m,
\end{array}\right. \\
& \leq \frac{x+1}{x^{\nu}+x^{1-\nu}} \\
& \leq\left\{\begin{array}{l}
\frac{m+1}{m^{\nu}+m^{1-\nu}} \text { if } M<1, \\
\max \left\{\frac{m+1}{m^{\nu}+m^{1-\nu}}, \frac{M+1}{M^{\nu}+M^{1-\nu}}\right\} \text { if } m \leq 1 \leq M, \\
\frac{M+1}{M^{\nu}+M^{1-\nu}} \text { if } 1<m,
\end{array}\right.
\end{aligned}
$$

which implies that

$$
\begin{aligned}
& \frac{x+1}{2} \times\left\{\begin{array}{l}
\frac{m^{\nu}+m^{1-\nu}}{m+1} \text { if } M<1, \\
\min \left\{\frac{m^{\nu}+m^{1-\nu}}{m+1}, \frac{M^{\nu}+M^{1-\nu}}{M+1}\right\} \text { if } m \leq 1 \leq M, \\
\frac{M^{\nu}+M^{1-\nu}}{M+1} \text { if } 1<m
\end{array}\right. \\
& \leq \frac{x^{\nu}+x^{1-\nu}}{2} \\
& \leq \frac{x+1}{2} \times\left\{\begin{array}{l}
\frac{M^{\nu}+M^{1-\nu}}{M+1} \text { if } M<1, \\
1 \text { if } m \leq 1 \leq M, \\
\frac{m^{\nu}+m^{1-\nu}}{m+1} \text { if } 1<m,
\end{array}\right.
\end{aligned}
$$

namely

$$
\phi_{\nu}(m, M) \frac{x+1}{2} \leq \frac{x^{\nu}+x^{1-\nu}}{2} \leq \Phi_{\nu}(m, M) \frac{x+1}{2}
$$

for any $x \in(0, \infty)$ and $\nu \in(0,1) \backslash\left\{\frac{1}{2}\right\}$.

Using the continuous functional calculus, we have for any operator $X$ with $m I \leq X \leq M I$ that

$$
\phi_{\nu}(m, M) \frac{X+I}{2} \leq \frac{X^{\nu}+X^{1-\nu}}{2} \leq \Phi_{\nu}(m, M) \frac{X+I}{2}
$$

for any $\nu \in(0,1) \backslash\left\{\frac{1}{2}\right\}$. 
Now, if we multiply both sides of (2.2) by $A^{-1 / 2}$ we have $m I \leq A^{-1 / 2} B A^{-1 / 2} \leq$ $M I$ and by writing the inequality (3.5) for $X=A^{-1 / 2} B A^{-1 / 2}$ we get

$$
\begin{aligned}
\phi_{\nu}(m, M) \frac{A^{-1 / 2} B A^{-1 / 2}+I}{2} & \leq \frac{\left(A^{-1 / 2} B A^{-1 / 2}\right)^{\nu}+\left(A^{-1 / 2} B A^{-1 / 2}\right)^{1-\nu}}{2} \\
& \leq \Phi_{\nu}(m, M) \frac{A^{-1 / 2} B A^{-1 / 2}+I}{2}
\end{aligned}
$$

for any $\nu \in(0,1) \backslash\left\{\frac{1}{2}\right\}$.

Finally, if we multiply both sides of (3.6) with $A^{1 / 2}$, then we get the desired result (3.4).

Finally, we have:

Corollary 3.3. Let $A, B$ be two positive operators. For positive real numbers $m, m^{\prime}, M, M^{\prime}$, put $h:=\frac{M}{m}, h^{\prime}:=\frac{M^{\prime}}{m^{\prime}}$ and let $\nu \in(0,1) \backslash\left\{\frac{1}{2}\right\}$. If either of the following conditions

(i) If $0<m I \leq A \leq m^{\prime} I<M^{\prime} I \leq B \leq M I$,

(ii) If $0<m I \leq B \leq m^{\prime} I<M^{\prime} I \leq A \leq M I$,

hold, then

$$
\frac{h^{\nu}+h^{1-\nu}}{h+1} A \nabla B \leq H_{\nu}(A, B) \leq \frac{\left(h^{\prime}\right)^{\nu}+\left(h^{\prime}\right)^{1-\nu}}{h^{\prime}+1} A \nabla B .
$$

\section{REFERENCES}

[1] S. S. Dragomir, Some new reverses of Young's operator inequality, Preprint $R G M I A$ Res. Rep. Coll. 18: Art. 130, 2015. [http://rgmia.org/papers/v18/v18a130.pdf] .

[2] S. S. Dragomir, On new refinements and reverses of Young's operator inequality, Preprint RGMIA Res. Rep. Coll. 18: Art. 135, 2015. [http://rgmia.org/papers/v18/v18a135.pdf] .

[3] S. S. Dragomir, Some inequalities for operator weighted geometric mean, Preprint RGMIA Res. Rep. Coll. 18: Art. 139, 2015. [http://rgmia.org/papers/v18/v18a139.pdf ] .

[4] S. S. Dragomir, Refinements and reverses of Hölder-McCarthy operator inequality, Preprint RGMIA Res. Rep. Coll. 18: Art. 143, 2015. [http://rgmia.org/papers/v18/v18a143.pdf] .

[5] S. S. Dragomir, Some reverses and a refinement of Hölderoperator inequality, Preprint RGMIA Res. Rep. Coll. 18: Art. 147, 2015. [http://rgmia.org/papers/v18/v18a147.pdf] .

[6] S. S. Dragomir, Some inequalities for Heinz operator mean, Preprint RGMIA Res. Rep. Coll. 18: Art. 163, 2015. [Online http://rgmia.org/papers/v18/v18a163.pdf] .

[7] S. S. Dragomir, Further inequalities for Heinz operator mean, Preprint RGMIA Res. Rep. Coll. 18: Art. 167, 2015. [Online http://rgmia.org/papers/v18/v18a167.pdf] .

[8] S. Furuichi, On refined Young inequalities and reverse inequalities, J. Math. Inequal. 5: 21-31, 2011.

[9] S. Furuichi, Refined Young inequalities with Specht's ratio, J. Egyptian Math. Soc. 20 : 46-49, 2012. 
[10] F. Kittaneh and Y. Manasrah, Improved Young and Heinz inequalities for matrix, $J$. Math. Anal. Appl. 361: 262-269, 2010.

[11] F. Kittaneh and Y. Manasrah, Reverse Young and Heinz inequalities for matrices, Lin. Multilin. Alg., 59: 1031-1037, 2011.

[12] F. Kittaneh, M. Krnić, N. Lovričević and J. Pečarić, Improved arithmetic-geometric and Heinz means inequalities for Hilbert space operators, Publ. Math. Debrecen, 80(34): 465-478, 2012.

[13] M. Krnić and J. Pečarić, Improved Heinz inequalities via the Jensen functional, Cent. Eur. J. Math. 11 (9): 1698-1710, 2013.

[14] F. Kubo and T. Ando, Means of positive operators, Math. Ann. 264: 205-224, 1980.

[15] W. Liao, J. Wu and J. Zhao, New versions of reverse Young and Heinz mean inequalities with the Kantorovich constant, Taiwanese J. Math. 19(2): 467-479, 2015.

[16] W. Specht, Zer Theorie der elementaren Mittel, Math. Z. 74:91-98, 1960.

[17] M. Tominaga, Specht's ratio in the Young inequality, Sci. Math. Japon., 55: 583-588, 2002.

[18] G. Zuo, G. Shi and M. Fujii, Refined Young inequality with Kantorovich constant, J. Math. Inequal., 5: 551-556, 2011.

(Silvestru Sever Dragomir) Mathematics, College of Engineering \& Science, Victoria University, PO Box 14428, Melbourne City, MC 8001, Australia.

Email address: sever.dragomir@vu.edu.au 